\title{
Critical frontier of the triangular Ising antiferromagnet in a field
}

\author{
Xiaofeng Qian, ${ }^{1}$ Maarten Wegewijs, ${ }^{2}$ and Henk W. J. Blöte ${ }^{1,3}$ \\ ${ }^{1}$ Lorentz Institute, Leiden University, P.O. Box 9506, 2300 RA Leiden, The Netherlands \\ ${ }^{2}$ Institut für Theoretische Physik, RWTH Aachen, 52056 Aachen, Germany \\ ${ }^{3}$ Faculty of Applied Sciences, Delft University of Technology, P.O. Box 5046, 2600 GA Delft, The Netherlands
}

(Received 27 November 2003; published 31 March 2004)

\begin{abstract}
We study the critical line of the triangular Ising antiferromagnet in an external magnetic field by means of a finite-size analysis of results obtained by transfer-matrix and Monte Carlo techniques. We compare the shape of the critical line with predictions of two different theoretical scenarios. Both scenarios, while plausible, involve assumptions. The first scenario is based on the generalization of the model to a vertex model, and the assumption that the exact analytic form of the critical manifold of this vertex model is determined by the zeroes of an $\mathrm{O}(2)$ gauge-invariant polynomial in the vertex weights. However, it is not possible to fit the coefficients of such polynomials of orders up to 10 , such as to reproduce the numerical data for the critical points. The second theoretical prediction is based on the assumption that a renormalization mapping exists of the Ising model on the Coulomb gas, and analysis of the resulting renormalization equations. It leads to a shape of the critical line that is inconsistent with the first prediction, but consistent with the numerical data.
\end{abstract}

DOI: 10.1103/PhysRevE.69.036127

PACS number(s): 05.50.+q, 64.60.Ak, 64.60.Cn, 64.60.Fr

\section{INTRODUCTION}

The triangular Ising model with equal nearest-neighbor coupling $K$ in a magnetic field has the reduced Hamiltonian

$$
\mathcal{H} / k_{B} T=-K \sum_{\langle i, j\rangle} s_{i} s_{j}-H \sum_{k} s_{k}
$$

where $s_{i}= \pm 1$, and $\langle i, j\rangle$ indicates summation over all pairs of nearest-neighbor sites. According to the exact solution by Houtappel [1] of the triangular Ising model in the absence of a magnetic field, the antiferromagnetic model has no phase transition at nonzero temperatures. The ground state is characterized by the condition that every elementary triangle contains spins of different signs. This constraint still leaves a considerable degeneracy, to such an extent that the zerotemperature antiferromagnet has a nonzero entropy. The ground state appears to have interesting properties. It is a critical state as shown by exact calculations [2] of the spinspin correlation function which appears to decay as a power law of the distance. A nonzero temperature $T>0$ destroys the critical state: the correlations then decay exponentially. However, for sufficiently low $T$, a sufficiently strong field $H>0$ induces a phase transition to a long-range ordered state, where the minus spins condense on one of the three sublattices. As noted by Alexander [3], the threefold symmetry of the ordered phase indicates that the transition belongs to the three-state Potts universality class. The nature of the transition was confirmed by Kinzel and Schick [4], using phenomenological scaling [5] and numerical transfer-matrix calculations; see also Noh and Kim [6] and Tamashiro and Salinas [7].

The critical line covers an infinite range of $K<0$ and $H$. A preview of our numerical data is given in Fig. 1. Since the phase diagram is symmetric in $H$, we restrict it to $H \geqslant 0$. For $K \rightarrow-\infty, H \rightarrow \infty$ while $6 K+H$ remains finite, the model maps [8] onto Baxter's hard-hexagon lattice gas of which the critical exponents are exactly known, and they do indeed fit the three-state Potts universality class. The asymptotic form of the critical line in this lattice-gas (LG) limit is

$$
K_{c}(H) \simeq-\frac{1}{6} H-\frac{1}{12} \ln \zeta_{c}, \quad \zeta_{c}=\frac{11+5 \sqrt{5}}{2},
$$

where $\zeta_{c}$ is the exact critical fugacity calculated by Baxter [9].

The critical line also extends to $K \rightarrow-\infty$ at small fields $|H|$. The behavior of the critical line in this limit has attracted attention because of the above peculiar ground-state properties, and the associated analytical and computational difficulties. It has been conjectured [4] that the critical line comes in vertically in the $1 / K$ versus $H / K$ diagram. In other words, when the Ising temperature goes to zero, also the reduced critical field $H_{c}$ (which includes a factor $1 / k_{B} T$ ) was supposed to go to zero. However, Nienhuis et al. [10] provided evidence that $H_{c}$ instead approaches a nonzero con-

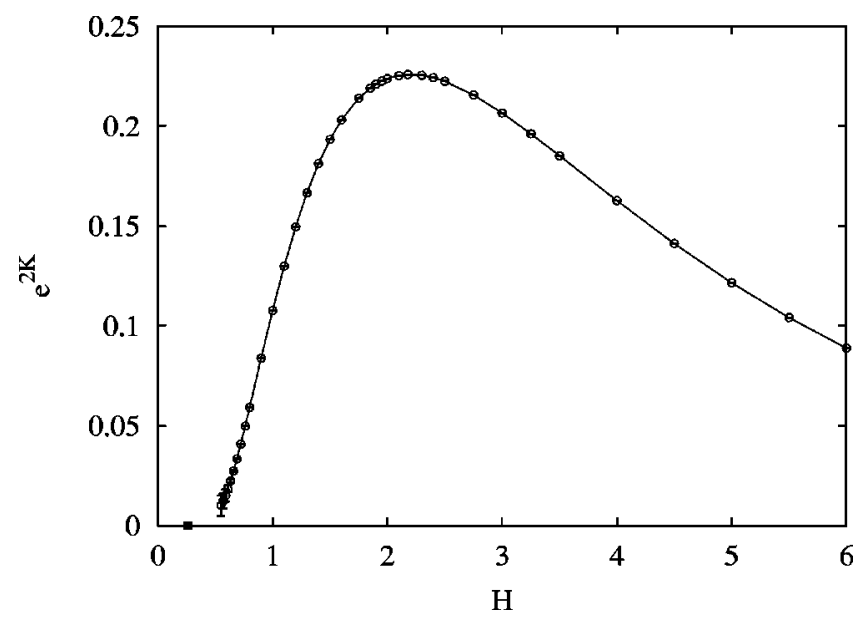

FIG. 1. Numerical results for the $\left(H, e^{2 K}\right)$ phase diagram. The circles denote the data points for Ising temperatures $T>0$ and the square the so-called KT point at $T=0$. 
stant when $K \rightarrow-\infty$. This result is based on an exact mapping of the zero-temperature Ising model on a solid-on-solid (SOS) model [11]. Using renormalization arguments, Nienhuis et al. obtained several critical exponents associated with physical fields. It was found that the reduced magnetic field is irrelevant: it does not immediately destroy the critical state at $K=-\infty$.

This renormalization analysis is not rigorous but still convincing. Several of its predictions agree with exact calculations [12] at $H=0$. The renormalization picture has been extended to include a nonzero field $H$ and Ising temperature $T$, as well as next-nearest-neighbor interactions [10]. It predicts that for $T=0$ the model undergoes an infinite-order transition to a long-range ordered phase at a finite value of the field $H$. In the SOS language this is a roughening transition, in the universality class of the Kosterlitz-Thouless (KT) transition [13]. The character of this transition was confirmed $[12,14]$ and located at $H_{\mathrm{KT}}=0.266(10)$ by means of transfermatrix calculations and phenomenological renormalization [12]. The associated finite-size-scaling analysis is problematic because of slow convergence due to logarithmic corrections at the KT transition point. Such corrections are possibly a reason why an analysis by de Queiroz et al. [15], without such corrections, yielded a result that is not fully consistent, namely, $H_{\mathrm{KT}}=0.211$ (7) (for the correct interpretation of this result it is essential that the field $H$ used in Fig. 1 and Table I of Ref. [15] does not contain a factor $1 / T$ [16]).

The estimated critical field $H_{\mathrm{KT}}$ at $T=0$ appears to be much smaller than estimates obtained at $T>0$. The question thus arises whether the Potts critical line for $T>0$ connects to the KT point at $T=0$. It is noteworthy that the renormalization scenario given in Ref. [10], which includes nextnearest-neighbor interactions $K_{\mathrm{NNN}}$, implies that the line of phase transitions limiting the ordered phase in the $\left(K_{\mathrm{NNN}}, T\right)$ plane does not connect to the transition line in the $\left(K_{\mathrm{NNN}}, H\right)$ plane. Thus one may ask the same question for the $\left(K_{\mathrm{NNN}}, H\right)$ and the $(H, T)$ plane. An answer to this question is provided by renormalization arguments presented in Sec. III. This approach also predicts the analytical form of the Potts critical line for $T \rightarrow 0$ while $H$ remains finite.

A different approach to find the shape of the critical line of an antiferromagnetic Ising model in a field was formulated by $\mathrm{Wu}[17]$ who noted that these models can be mapped on vertex models, and that these vertex models have symmetry properties that impose restrictions on the analytic form of their critical manifolds. He also noted that the critical manifolds of the exactly solved vertex models are determined by the zeroes of homogeneous polynomials in the vertex weights that are invariant under the symmetry group of the model. On the basis of the assumption that the latter form of the critical subspace also applies to vertex models that are equivalent with antiferromagnetic Ising models in a field [18], one may thus attempt to solve for the unknown independent coefficients of the homogeneous polynomial, the number of which is dramatically reduced by symmetry restrictions. In actual applications, the number of equations is still not enough to solve all unknown coefficients, and additional numerical input is required, for instance, from a numerical transfer-matrix analysis.
This approach is more ambitious than the renormalization analysis in the sense that its aim is to describe the whole critical manifold. It has been applied to the Ising antiferromagnets on the honeycomb lattice [19] and on the square lattice [20]. The transfer-matrix data, with accuracies in the order of $10^{-10}$, could be successfully described by such invariant polynomials of relatively low order. Nevertheless one may remark that these analyses did not provide solid evidence for the exact form of the critical line of the Ising antiferromagnet.

Application of this approach to the triangular Ising model leads to some additional complications. First, the topology of the phase diagram is less simple, which relates to the fact that the lattice is not bipartite. Second, the three-state Potts character of the critical line implies that corrections-toscaling converge less well in comparison with the Ising case, so that it is not feasible to reach the same degree of numerical accuracy.

In this paper we compare the results of both theoretical approaches to our numerical data for the triangular Ising antiferromagnet. In Sec. II we formulate the invariantpolynomial scenario and derive an exact restriction on the critical line which must hold if this line is analytic in the KT point. We explicitly construct invariant polynomials of arbitrary order in the Ising vertex weights whose roots exhibit this behavior. A summary of the Coulomb gas scenario and an analysis of the renormalization-flow equations follows in Sec. III. The analytic forms of the critical lines predicted by these two scenarios appear to be mutually inconsistent for $T \rightarrow 0$ at finite $H$. In Sec. IV we outline our transfer-matrix construction and present accurate results for the critical points. This section also includes a Monte Carlo analysis of the critical amplitudes. An analysis and a discussion of these results is given in Sec. V. Finally, we draw our conclusions in Sec. VI.

\section{O(2) INVARIANT POLYNOMIALS IN THE VERTEX WEIGHTS}

The mapping of the triangular Ising model on the 64vertex model involves the introduction of bond variables $b_{i j}=0$ or 1 between nearest-neighbor spins and summation over the Ising variables [17]. Since the bond variables are independent, there are $2^{6}=64$ distinct vertices. But these turn out to have only seven distinct weights

$$
W_{s_{1} \cdots s_{6}}=W_{s}=h^{s \bmod 2} z^{s / 2}
$$

where $h=\tanh (H)$ and $z=\tanh (K)$. These weights are "symmetric," i.e., depend only on the number of covered bonds $\sum_{j}{ }^{\mathrm{NN}} b_{i j}$ connecting a vertex $i$ to its six nearest neighbors $j$. The weights can take imaginary ( $s=$ odd, $K<0$ ) or real (otherwise) values. The partition function of an $N$-site system is a homogeneous polynomial of order $N$ in the weights:

$$
Z_{\mathrm{vertex}} \equiv \sum_{\left\{b_{i j}\right\}} \prod_{k} W_{s_{1}(k) \cdots s_{6}(k)}
$$


Both pertinent indices of neighboring vertices $i$ and $j$ in this product are set equal to the bond variable $b_{i j}=0,1$. The summation runs over all possible configurations of the bond variables. The partition function of the Ising model differs from Eq. (4) only by a multiplicative factor which is nonsingular for finite $H$ and $K$ :

$$
Z_{\text {Ising }}(H, K)=2 \cosh (H)^{N} \cosh (K)^{(3 / 2) N} Z_{\text {vertex }}\left[\left\{W_{s}(h, z)\right\}\right] .
$$

A crucial property of Eq. (4) is that the summation over one of the bond variables $b_{i j}$ is invariant with respect to any $\mathrm{O}(2)$ transformation

$$
R(\theta, \varepsilon)=\left(\begin{array}{cc}
\cos \theta & -\varepsilon \sin \theta \\
\sin \theta & \varepsilon \cos \theta
\end{array}\right)
$$

with respect to the indices of the connected vertices. Here, $\operatorname{det}(R)=\varepsilon= \pm 1$ distinguishes the $\mathrm{SO}(2)$ subgroup of proper rotations $(\varepsilon=+1)$ from the improper transformations $(\varepsilon$ $=-1$ ) which also include a reflection. Application of this transformation to all bonds connecting neighboring vertices (assuming periodic boundary conditions) leads to a partition sum of the same form but with new weights

$$
W_{s_{1}^{\prime} \cdots s_{6}^{\prime}}^{\prime}=R_{s_{1}^{\prime} s_{1}}(\theta, \varepsilon) \cdots R_{s_{6}^{\prime} s_{6}}(\theta, \varepsilon) W_{s_{1} \cdots s_{6}},
$$

where we use the dummy summation convention $s_{i}=0,1$. This gauge transformation preserves the symmetry mentioned under Eq. (3). However, only special O(2) transformations preserve the Ising weight parametrization expressed by the right-hand side of Eq. (3). A trivial example is the reflection $\theta=0, \varepsilon=-1$ effecting $W_{s}(h, z) \rightarrow W_{s}(-h, z)$. This corresponds to an external field inversion $H \rightarrow-H$. Below we will first discuss another less trivial transformation which also leads to weights of the Ising form (3) up to a common factor. This will have consequences for the asymptotic behavior of the critical curve $h_{c}(z)$. In order to investigate the assumption that the critical curve is a root of an $\mathrm{O}(2)$ invariant homogeneous polynomial in the vertex weights, we explicitly construct these polynomials of arbitrary order. Conclusions about their compatibility with our numerical data will be drawn in Sec. V.

\section{A. Dual transformation}

The $\mathrm{O}(2)$ transformation $\theta=\pi / 2, \varepsilon=\mp 1$ changes the weights as $W_{s}(h, z) \rightarrow z^{3} W_{s}( \pm \operatorname{sgn}(z) h, 1 / z)$. This connects (up to a nonsingular factor) the Ising weights (3) for physical values of $|h|,|z| \leqslant 1$ to weights of the same form but with unphysical values $|h|,|z| \geqslant 1$ corresponding to complex fields $H \pm i \pi / 2, K \pm i \pi / 2$. Extending the vertex model with weights (3) to all real values of $h, z$ the antiferromagnetic $(z<0)$ critical curve of this model $h_{c}(z)$ has a physical and nonphysical branch which are connected by the dual transformation

$$
(h, z) \rightarrow(h, 1 / z)
$$

and which is separated by the self-dual LG and KT points at $z=-1$. This is a rigorous result of seemingly little use. However, we will now demonstrate that, combined with the assumption that the critical curve is analytical for $z=-1$, this severely constrains the shape of the antiferromagnetic branches of the critical curve for physical values of $h, z$. Consider the derivatives of a branch of the critical line $h_{c}(z)$ at $z=-1$, assuming it is analytical there. Differentiating the constraint $h_{c}(z)=h_{c}(1 / z) n$ times and recursively solving for the derivatives $h_{c}^{(k)}=\partial^{k} h_{c} /\left.\partial z^{k}\right|_{z=-1}$ up to order $k$ $=1, \ldots, n$ one finds that each odd order derivative can be expressed as a linear combination of lower even order derivatives with integral coefficients. The expansion of $h_{c}(z)$ must take the form

$$
h_{c}=h_{c}^{(0)}+\frac{1}{2 m !} h_{c}^{(2 m)}\left[(1+z)^{2 m}+m(1+z)^{2 m+1}\right]+\cdots
$$

Here $m \geqslant 1$ is some integral number, i.e., the first nonvanishing term is of even order. A notable feature of Eq. (9) is that, if the critical line is analytical at $z=-1$, it must satisfy $d h_{c} / d z=0$. We now consider the implications for the KT and LG asymptotic lines. First, the LG asymptotic relation Eq. (2) expressed in the variables $h, z$ reads

$$
\frac{1+h}{1-h}=\frac{1}{\zeta_{c}}\left(\frac{1-z}{1+z}\right)^{6}
$$

which indicates analyticity of the exact form of the LG branch of the critical line at $z=-1$. The first two nonvanishing terms in the resulting expansion

$$
h_{c}(z)=1-\frac{\zeta_{c}}{2^{5}}\left[(1+z)^{6}+3(1+z)^{7}+\cdots\right]
$$

are the same as in the expansion of Eq. (10) with $m=6$. We note that the LG asymptotic curve Eq. (10) is invariant under $(h, z) \rightarrow(h, 1 / z)$ up to all orders. This is due to the dual symmetry of the corresponding vertex model. Secondly, the approach of the critical curve to the KT asymptotic line must also be of the form (9). However, the integer value $m \geqslant 1$ in this case is unknown. Expressed in the physical variables $H, K$, we find a logarithmic divergence

$$
K(H)=\frac{1}{4 m} \ln \left(H_{c}-H_{\mathrm{KT}}\right)+\text { const. }
$$

This is the central result of this section. We emphasize that it is based on the assumption that the KT branch of critical line is analytic at $z=-1$. Roots of $\mathrm{O}(2)$ invariant polynomial equations, which we construct explicitly below, all have this property and in the general case $m=1$.

\section{B. $O(2)$ invariant polynomials in the Ising weights}

Now we explicitly construct homogeneous polynomial equations in the Ising weights which incorporate all the constraints imposed by the $\mathrm{O}(2)$ gauge symmetry (including 
those discussed above). Following Perk et al. [21] we change to the eigenbasis of the $\mathrm{SO}(2)$ subgroup of rotations $(\varepsilon=+1)$ via the $2 \times 2$ matrix $\alpha_{s_{i} \sigma_{i}}=\frac{1}{2}\left(-i \sigma_{i}\right)^{s_{i}}$ :

$$
W_{s_{1} \cdots s_{6}}=\alpha_{s_{1} \sigma_{1}} \cdots \alpha_{s_{6} \sigma_{6}} A_{\sigma_{1}} \cdots \sigma_{6} .
$$

Note that $s_{i}=0,1$ but $\sigma_{i}= \pm 1$. In this basis the transformation Eq. (7) takes the simple explicit form $(\varepsilon= \pm 1)$ :

$$
A_{\sigma_{1} \cdots \sigma_{6}}^{\prime}=e^{i \theta\left(\sigma_{1}+\cdots+\sigma_{6}\right)} A_{\varepsilon \sigma_{1} \cdots \varepsilon \sigma_{6}} .
$$

For the symmetric vertex model, the seven components transform as $A_{\sigma}^{\prime}=e^{i \theta \sigma_{1}} A_{\varepsilon \sigma}$, where $\sigma=\sigma_{1}+\cdots+\sigma_{6}=0$, $\pm 2, \pm 4, \pm 6$. In this basis the Ising weights are complex:

$$
\begin{aligned}
A_{2 k}= & (z+1)^{3-k}\left[\sum_{m=0}^{k}\left(\begin{array}{c}
2 k \\
2 m
\end{array}\right)(-z)^{m}\right. \\
& \left.+i h \sqrt{z} \sum_{m=0}^{k-1}\left(\begin{array}{c}
2 k \\
2 m+1
\end{array}\right)(-z)^{m}\right],
\end{aligned}
$$

where $k=0,1,2,3$ and $A_{-2 k}=A_{2 k}^{*}$. Invariant polynomials which transform with parity \pm 1 (i.e., $I_{ \pm} \rightarrow \pm I_{ \pm}$) have the simple form $I_{ \pm}=\frac{1}{2}\left(\Pi_{\sigma} A_{\sigma}^{n_{\sigma}} \Pi_{\sigma} A_{\sigma}^{n_{-\sigma}}\right)$ (or a linear combination of these) with exponents which satisfy $\Sigma_{\sigma} \sigma n_{\sigma}=0$. A minimal finite set of such exponents can be found which generate all other solutions by linear combination with integer coefficients. This implies that any $\mathrm{O}(2)$ invariant $I_{ \pm}$can be generated as a polynomial function of a minimal set of so-called fundamental invariants. These 14 polynomials have been constructed in Ref. [21]. The crucial point is to eliminate all dependencies due to polynomial relations between the fundamental invariants (called syzygies), and further dependencies introduced by the parametrization (15). To generate invariants of parity +1 we need to retain only four fundamental $\mathrm{O}(2)$ invariants of parity +1 which can be compactly be written in variables $s=1-h^{2}=1 / \cosh ^{2} H$ and $u=1+z=e^{K} / \cosh K$

$$
\begin{aligned}
& I_{0}=A_{0}=u^{3}, \\
& I_{k}=A_{2 k} A_{-2 k}=u^{6}+u^{6-2 k} \Omega_{k}(u) s,
\end{aligned}
$$

where

$$
\begin{aligned}
& \Omega_{1}(u)=4(1-u), \\
& \Omega_{2}(u)=4(1-u)(2 u-4)^{2}, \\
& \Omega_{3}(u)=4(1-u)(3 u-4)^{2}(u-4)^{2} .
\end{aligned}
$$

The most general invariant polynomial of even order $2 e$ $\geqslant 2$ and parity +1 in the Ising weights is

$$
f_{2 e}=\sum_{j=0}^{e} I_{0}^{2(e-j)}\left[\sum_{l=0}^{j} c_{l}^{j} I_{2}^{l} I_{3}^{j-l}+\sum_{l=1}^{j} c_{j+l}^{j} I_{1}^{l} I_{2}^{j-l}\right] .
$$

Cross terms of $I_{1}$ and $I_{3}$ have been eliminated using a polynomial relation. The $(e+1)^{2}$ coefficients in Eq. (18) correspond 1-to-1 to an invariant expression in the Ising weights of order $2 e$. To exclude a trivial factorization to an even order polynomial, i.e., $f_{2 e}=I_{0}^{2} f_{2(e-1)}$, we require $c_{0}^{0} \neq 0$. Polynomial invariants of odd order and parity +1 can be shown to factorize trivially, $f_{2 e+1}=I_{0} f_{2 e}$, and thus need not be considered further. Finally, polynomial invariants of any order and parity -1 can also be discarded. We find that for $z=-1$ such polynomials have no other root than $h= \pm 1$, i.e., the KT point cannot be described. The expansion of $f_{2 e}$ in Eq. (18) is not well suited to impose restrictions on the coefficients from known properties of the critical curve of the triangular lattice Ising model. A more convenient but equivalent expansion is obtained by replacing $I_{k} \rightarrow I_{k}-I_{0}^{2}, k$ $=1,2,3$ and $c_{l}^{j} \rightarrow \kappa_{l}^{j}$ in Eq. (18). This gives the final explicit form

$$
\begin{aligned}
f_{2 e}= & \sum_{j=0}^{e}\left[\sum_{l=0}^{j} \kappa_{l}^{j} \Omega_{2}^{l} \Omega_{3}^{j-l} u^{2 l}\right. \\
& \left.+\sum_{l=1}^{j} \kappa_{j+l^{j}}^{j} \Omega_{1}^{l} \Omega_{2}^{j-l} u^{2 l+2 j}\right] u^{6(e-j)} s^{j} .
\end{aligned}
$$

Since $\Omega_{k}(u) \rightarrow 4^{2 k-1}$ in the KT $\left(u \rightarrow 0, s=1-h_{\mathrm{KT}}^{2} \neq 0\right)$ and LG $(s, u \rightarrow 0)$ limit we can easily solve $f_{2 e}(s, u)=0$ for the asymptotic relations between $s$ and $u$ in each limit. We find that consistent polynomials must satisfy $\kappa_{0}^{0}=-4^{3} \zeta_{c} \kappa_{0}^{1}$, where $\zeta_{c}$ is given by Eq. (2) (LG) and $\kappa_{0}^{e}=\kappa_{1}^{e}=\kappa_{2}^{e}=0(\mathrm{KT})$. This already excludes $f_{2}=0$ as a candidate. We thus have $(e+1)^{2}-5=4,11,20,31$ independent coefficients in a polynomial of order $2 e=4,6,8,10$, respectively. The asymptotic value of $s$ in the KT limit is determined by $s_{\mathrm{KT}}$ $=4 \kappa_{0}^{e-1} / \kappa_{3}^{e}$. This may be used to either extract this value after a fitting procedure or as an extra constraint. One can verify from the general form Eq. (19) that the approach to the KT value indeed takes the form Eq. (12) with $m=1$. This is dictated by the dual transformation property Eq. (8) combined with the analyticity of the branches determined by $f_{2 e}=0$. Higher integer values for $m$ are also possible but require certain coefficients in Eq. (19) to be strictly zero, which is not supported by the numerical data.

\section{RENORMALIZATION ANALYSIS}

\section{A. Mapping on the Gaussian model}

At zero temperature, the three spins of each elementary triangle cannot have the same sign. Thus each triangle has two bonds between antiparallel spins and one bond between parallel spins. When all bonds between parallel spins are erased, one obtains a lattice tiling with rhombi. This tiling can also be interpreted as a stack of cubes viewed from the $(1,1,1)$ direction. Thus the zero-temperature antiferromagnetic triangular Ising model is equivalent with a solid-onsolid (SOS) model [11]. The SOS model consists of height variables $h_{i}$ where $i$ denotes the lattice site. The height variables assume integer values satisfying $h_{i} \bmod 3=c_{i}$, where $c_{i}=0,1$ or 2 denotes the sublattice of site $i$. Apart from an infinite constant, the Hamiltonian becomes 


$$
\begin{aligned}
\mathcal{H} / k_{B} T= & K_{\infty} \sum_{\langle i, j\rangle}\left(1-\delta_{\left|h_{i}-h_{j}\right|, 1}\right)\left(1-\delta_{\left|h_{i}-h_{j}\right|, 2}\right) \\
& -H \sum_{k}\left(2 \delta_{\left(h_{k} \bmod 2,0\right)}-1\right)
\end{aligned}
$$

where we set $K_{\infty} \rightarrow \infty$ so that the product $\left(1-\delta_{\left|h_{i}-h_{j}\right|, 1}\right)(1$ $-\delta_{\left.\left|h_{i}-h_{j}\right|, 2\right)}$ restricts the height differences between nearestneighbor sites to 1 or 2 . The Kronecker delta in the second term counts the numbers of + spins.

The equivalence with the Ising model (1) makes it possible to express the height-height correlation function [10]

$$
g(r)=\left\langle\left[\left(h_{r}-h_{0}\right)-\left\langle h_{0}-h_{r}\right\rangle\right]^{2}\right\rangle
$$

in terms of Ising correlations. From the exact results for $K$ $=-\infty, H=0$, it follows [10] that

$$
g(r) \simeq \frac{9}{\pi^{2}} \ln (r)+\text { const }
$$

where $r$ is the distance between the correlated sites. This result is very useful in the context of a renormalization mapping by Nienhuis et al. [10] on the Gaussian model with Hamiltonian

$$
-\mathcal{H} / k_{B} T=\frac{2 \pi}{T_{R}} \sum_{\langle i, j\rangle}\left(h_{i}-h_{j}\right)^{2}+\sum_{p} S_{p} \sum_{i} \cos \frac{2 \pi h_{i}}{p},
$$

where the second summation contains so called spin-wave perturbations of the Gaussian model, i.e., a periodic potential acting on the Gaussian height variables. A term with $p$ $=1$ originates from the discreteness of the height variables in Eq. (20). A nonzero magnetic field $H$ favors triangles with only one minus spin; in the SOS model this leads to an energy alternation between even and odd heights. This maps on a spin-wave perturbation with $p=2$ in the Gaussian model.

The mapping of Eq. (20) to Eq. (23) is not exact, so that the renormalized temperature $T_{R}$ is in principle unknown. However, the height-height correlation function of the Gaussian model is known to depend on $T_{R}$ as

$$
g(r) \simeq \frac{T_{R}}{2 \pi^{2}} \ln (r)
$$

Comparison with Eq. (22) shows that

$$
T_{R}=18
$$

for $H=0$. Once $T_{R}$ is known, several quantities of interest can be calculated for the Gaussian model. These quantities include the scaling dimensions associated with the so-called spin-wave and vortex perturbations in the Gaussian model. Since an elementary excitation of the Ising model, i.e., a triangle with three equal Ising spins, leads to an SOS height mismatch of six units, the Ising temperature field $t_{I}$ is represented by the fugacity $V_{q}$ of the $q= \pm 6$ vortices. On the basis of the known results for the scaling dimensions of $S_{p}$ and $V_{q}$ in the Gaussian model [22], a number of properties of the triangular Ising model, including a part of the phase diagram extended in the direction of $T_{R}$, have been derived [10]. In this work we make use of the language of the Coulomb gas formulation [23] to express the relevant scaling dimensions. The appropriate parameters are the renormalized coupling constant $g_{R}$, and electric charges $e$ and magnetic charges $m$. Their relation with the parameters of the Gaussian model can be expressed as

$$
\begin{gathered}
g_{R}=36 / T_{R}, \\
e=6 / p, \\
m=q / 6 .
\end{gathered}
$$

In this language, the scaling dimensions $X_{e, m}$ associated with the activity of charges $(e, m)$ are

$$
X_{e, m}=\frac{e^{2}}{2 g_{R}}+\frac{g_{R} m^{2}}{2} .
$$

From Eq. (25) we see that $g_{R}=2$ for $H=0$. Since the Ising temperature field is associated with magnetic charges \pm 1 , the Ising temperature renormalization exponent $y_{t_{I}}=2$ $-X_{0,1}=1$ is relevant. The system is thus disordered for all $T>0$. However, the exponent $y_{h}=2-X_{3,0}=-1 / 4$ associated with the uniform magnetic field $H$ is irrelevant. Thus, at $T=0$ the system remains critical for a certain range of the Ising field $H$. In the renormalization scenario outlined in Ref. [10] a phase transition to the long-range ordered phase occurs when $H$ grows large enough. In the SOS language this ordered phase is flat, and transition is of the "roughening" type, by duality related to the Kosterlitz-Thouless phase transition [13].

\section{B. Renormalization flow}

For an analysis of the renormalization flow at nonzero magnetic field $H$ it is necessary to include the Coulomb gas coupling $g_{R}$ because the field, although irrelevant at $g_{R}=2$, tends to suppress height differences, i.e., to increase $g_{R}$. As deduced in Ref. [10] such an effect can also be realized without breaking the Ising symmetry, by the introduction of ferromagnetic next-nearest-neighbor interactions into the model (1). Although we restrict our numerical investigation to the two-parameter model (1), the renormalization analysis still requires a set of three nonlinear scaling fields, which are chosen as

$$
\begin{gathered}
t=\frac{9}{2 g_{R}}-2, \\
h=\alpha_{1} H+\alpha_{3} H^{3}+\cdots, \\
t_{I}=e^{2 K}+\cdots
\end{gathered}
$$

The constant $\alpha_{1}$ determines the scale of $t$ which remains to be determined. Apart from that, the expansion coefficients $\alpha_{j}$ 
are in principle unknown. The Ising temperature field $t_{I}$ is in lowest order chosen as the Boltzmann factor of an elementary Ising excitation, i.e., a triangle with three equal spins. It thus describes the activity of the magnetic charges $m= \pm 1$ in the Coulomb gas. In order to describe the renormalization of these parameters in the immediate vicinity of the fixed line $h=t_{I}=0$, the following renormalization exponents apply:

$$
\begin{gathered}
y_{t}=0, \\
y_{h}=2-\frac{9}{2 g_{R}}, \\
y_{t_{I}}=2-\frac{g_{R}}{2} .
\end{gathered}
$$

First we address the special case $T=t_{I}=0$. Because of the marginality of $t$ we add a nonlinear term in the flow for $t$. In differential form, the equations become

$$
\frac{d h(l)}{d l}=-h t, \quad \frac{d t(l)}{d l}=-h^{2},
$$

where $l$ parametrizes the rescaling factor $b$ as $b=\exp (l)$. In principle one has an unknown amplitude in the second equation, but we have disposed of it by a proper choice of $\alpha_{1}$. Thus, the scale of $t$ is set such as to simplify the renormalization equations to Eq. (30). The equation for $h$ follows from the usual form $h^{\prime}=b^{y_{h}} h$ after substitution of $b$ in terms of $l$, of $y_{h}$ using Eq. (29), and $g_{R}$ in terms of $t$. The sign in the equation for $t$ follows because $h$ suppresses the height differences in the SOS language. The flow equations (30) are equivalent to those describing the Kosterlitz-Thouless [13] and roughening transitions. Elimination of $l$ from Eq. (30) and integration yields the trajectory in the $h, t$ plane as

$$
h^{2}=t^{2}+c^{2}
$$

where the constant $c$ follows from the initial conditions which are chosen as $h(0)=1 / 4+\delta h, t(0)=1 / 4$. The physical motivation of this choice is that the KT transition line obeys $h=t$, so that we select a point at a distance $\delta h$ to the KT point of the nearest-neighbor model Eq. (1). For small $\delta h$ one finds $c^{2} \approx \delta h / 2$. Elimination of $h$ in Eq. (30) leads to

$$
\frac{d t(l)}{d l}=-t^{2}-c^{2}
$$

Integration, substitution of the initial conditions, and some rearrangement lead to the renormalization flow for $t_{I}=0$ as

$$
t=\frac{\sqrt{\delta h / 2}}{\tan (\sqrt{8 \delta h}+l \sqrt{\delta h / 2})} .
$$

Next we introduce a nonzero Ising temperature $T$ so that also $t_{I}>0$. It seems reasonable to assume that the renormalization flow of $h$ and $t$ is not seriously affected for small $t_{I}$. For simplicity we make a stronger assumption, namely that the flow of $h$ and $t$ is independent of that of $t_{I}$. We first focus on the question whether the three-state Potts critical line in the $(H, T)$ plane extends to the KT transition at $T=0$, or, in other words, whether there are points in the immediate vicinity of $\left(H=H_{\mathrm{KT}}, T=0\right)$ that flow towards a region where we can be confident that a Potts-like transition occurs. For small $\delta h / 2$ and $t_{I}$ the first part of the path will be governed by the KT fixed point. We assume that the flow will bring the system to a boundary which separates the regions governed by the KT and Potts fixed points. This boundary is obviously not determined in a quantitative sense, but this does not matter for the present scaling argument. Let it be sufficient to define this region by requiring that $h$ and $t_{I}$ reach values of order 1 .

We search for this region by choosing the (somewhat arbitrary) renormalized temperature $g_{R}=3$ where we have evidence [24] that the Potts transition connects to the neighborhood of $T=H=0$. The shape of the critical line at $g_{R}=3$ is determined by the flow equations for $t_{I}$ and $h$, namely,

$$
\begin{gathered}
t_{I}(b)=b^{y_{t}} t_{I}, \\
h(b)=b^{y_{h}} h,
\end{gathered}
$$

where one may take $y_{t_{I}}=y_{h}=1 / 2$ as long as $t_{I}$ and $h$ are small so that the change of $g_{R}$ can be neglected. Then, $t_{I}(b) / h(b)$ is constant along a flow line and can be chosen such that the model is critical, say for

$$
t_{I}(b) / h(b)=\beta \text {, }
$$

where $\beta$ is a constant of order 1 . For larger values of $t_{I}$ and $h$ the relation will no longer be linear but it is reasonable to expect, and in agreement with numerical results [24,25], that there is a fair range where $\beta$ is still of order 1 .

Thus we consider a point $h(0)=1 / 4+\delta h, t(0)=1 / 4$, $t_{I}(0)=\delta t_{I}$ in the vicinity of the KT point and apply a transformation such that the system flows to $g_{R}=3$ or $t=-1 / 2$. According to Eq. (33) the scale factor of this transformation is $b=\exp (l)=\exp (\pi \sqrt{2 / \delta h})$. It follows from Eq. (33) that (for small $\delta h$ and $\delta t_{I}$ ) the system is located near the KT fixed point $h=0, t=0$ for most of the range of $l$. Therefore, the flow of the Ising temperature field $t_{I}$ is determined by the exponent $y_{t_{I}}=7 / 8$ at $g_{R}=9 / 4$ or $t=0$. Thus, at $g_{R}=3$ it reaches the value $t_{I}=b^{7 / 8} \delta t_{I}=\exp (7 \pi / 4 \sqrt{2 \delta h}) \delta t_{I}$. Since $h$ $=-t=1 / 2$ up to unimportant corrections, Eq. (35) leads to

$$
\delta t_{I}=\frac{\beta}{2} \exp \left(-\frac{7 \pi}{4 \sqrt{2 \delta h}}\right)
$$

which solves $\delta t_{I}$ for all $\delta h>0$. This implies that the Potts critical sheet connects to the KT point. The resulting renormalization flow is sketched in Fig. 2. Substitution of Eq. (28) and $H=H_{\mathrm{KT}}+\delta H$ leads in lowest order to

$$
K \simeq \frac{1}{2}\left[\ln \frac{\beta}{2}-\frac{7 \pi}{4 \sqrt{2 \alpha\left(H-H_{\mathrm{KT}}\right)}}\right],
$$

where $\alpha$ is a function of the $\alpha_{j}$. This equation determines the shape of the critical line near the KT point of the model (1) and is clearly incompatible with the prediction of the invariant-polynomial scenario, Eq. (12). 


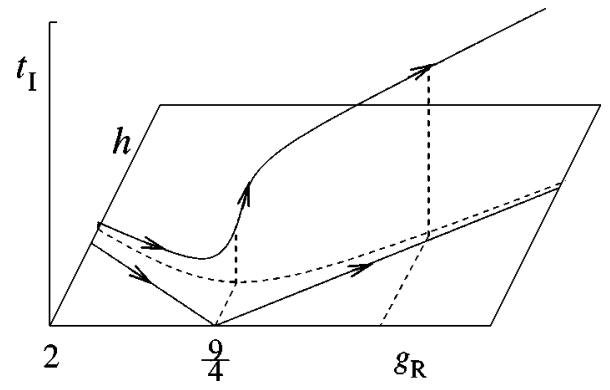

FIG. 2. Sketch of the renormalization flow in the parameter space of the renormalized coupling $g_{R}$, the scaling field $h$, and the Ising temperature field $t_{I}$. The flow of $g_{R}$ and $h$ is anomalously slow near the point $g_{R}=9 / 4, h=0$ : most of the growth of $t_{I}$ occurs here.

\section{NUMERICAL METHODS}

\section{A. Transfer-matrix calculations}

Most of the transfer-matrix calculations were performed for $T>0$ so that we had to use a binary representation for the Ising spins, leading to a transfer matrix of size $2^{L} \times 2^{L}$ for a system with finite size $L$. We define the spin lattice on the surface of a cylinder, whose axis determines the transfer direction. We have used two choices for the orientation of the lattice: one set of bonds parallel or perpendicular with respect to the axis. For the first case one may apply a decoration transformation to one half of the parallel bonds in order to construct a symmetric transfer matrix. However, the decoration of antiferromagnetic bonds leads to complex weights which we wish to avoid. We have thus used a nonsymmetric transfer matrix, in combination with a suitable tridiagonalization method to find the leading eigenvalues. These were obtained for even linear system sizes up to $L=22$, which corresponds with an actual finite size of $11 \sqrt{3}$ nearestneighbor bonds. The second construction, with a set of edges perpendicular to the transfer direction, leads to a symmetric matrix when two layers of spins are added. This allows the use of the conjugate-gradient method which is, in our applications, more stable than the tridiagonalization method. Finite-size calculations with $L$ multiples of 3 up to $L=24$ were performed using this second construction.

A sparse-matrix decomposition was used for both constructions. Most of the technique is already implicit in the work of Nightingale [5]. Further details are listed in Ref. [26] which concerns the case of the honeycomb lattice, but the essential steps are applicable to the triangular lattice as well. During the analysis of the results of both types of transfer matrix we found that they were mutually consistent. Furthermore it became clear that the second transfer matrix, with a set of bonds perpendicular to the transfer direction, allowed a somewhat more accurate analysis. In the following we describe the situation of the second construction.

For $T=0$ the transfer matrix decomposes in a number of diagonal submatrices characterized by a conserved number of "strings" so that the numerical diagonalization task simplifies. The transfer-matrix construction for this case has been outlined in Ref. [12] and enabled the study of systems with linear sizes up to $L=27$.
The magnetic correlation function along the coordinate $r$ in the length direction of the cylinder is defined as $g_{m}(r)$ $=\left\langle s_{0} s_{r}\right\rangle$. At large $r$, this correlation function decays exponentially with a characteristic length scale $\xi$ that depends on $K, H$, and $L$

$$
g_{m}(r) \propto e^{-r / \xi(K, H, L)}
$$

and can be calculated from the largest two eigenvalues $\lambda_{0}$ and $\lambda_{1}$ of the transfer matrix

$$
\xi^{-1}(K, H, L)=\frac{1}{\sqrt{3}} \ln \left(\lambda_{0} / \lambda_{1}\right),
$$

where the factor $\sqrt{3}$ is a geometric factor, i.e., the ratio between the thickness of two layers added by the transfer matrix and the length of a nearest-neighbor bond. The significance of these relations lies in the fact that the assumption of conformal invariance [27] links $\xi$ on the cylinder with the magnetic scaling dimension $X_{m}$ (one half of the magnetic correlation function exponent $\eta$ ). In terms of the scaled gap

$$
X_{m}(K, H, L) \equiv \frac{L}{2 \pi \xi(K, H, L)}
$$

one has $X_{m}(K, H, L) \simeq X_{m}$ in the limit of large $L$. Since the three-state Potts universal value of the magnetic scaling dimension is known to be $X_{m}=\frac{2}{15}$, and the transfer-matrix algorithm evaluates $X_{m}$ as a function of its arguments, one can find a numerical approximation to the critical value of $K$ for a given value of $H$ or vice versa. The shape of the critical line prescribes the use of different ways in different regions. For small $H$ and large $|K|$, the critical line is almost parallel to the zero-field line, so that it becomes more efficient to solve for $H$ than for $K$.

As a consequence of corrections to scaling, the solution will not precisely coincide with the critical point. The effects of an irrelevant scaling field $u$ and a small deviation $t$ with respect to the critical value of $H$ or $K$ are expressed by

$$
X_{m}(K, H, L)=X_{m}+a u L^{y_{i}}+b t L^{y_{t}}+\cdots,
$$

where $a$ and $b$ are unknown constants, $X_{m}=\frac{2}{15}, y_{i}=-\frac{4}{5}$, and $y_{t}=\frac{6}{5}$ for the three-state Potts universality class. Thus the solution for $K$ of

$$
X_{m}(K, H, L)=\frac{2}{15}
$$

which is denoted $K_{c}^{(1)}(H, L)$, depends on the finite size $L$ and the irrelevant field as

$$
K_{c}^{(1)}(H, L)=K_{c}+c_{1} L^{y_{i}-y_{t}}+\cdots
$$

because the two correction terms in Eq. (41) must cancel and $t \propto K_{c}{ }^{(1)}(H, L)-K_{c}$. We thus generated sequences of iterated estimates of $K_{c}$ by solving $K_{c}{ }^{(2)}(H, L)$ and $c_{1}(L)$ in the equations

$$
K_{c}^{(2)}(H, L)=K_{c}^{(1)}(H, l)+c_{1}(L) l^{y_{i}-y_{t}}
$$


TABLE I. Extrapolated results for selected points on the critical line.

\begin{tabular}{lccccc}
\hline \hline No. & $H$ & $K$ & No. & $H$ & $K$ \\
\hline 1 & $0.55(5)$ & -2.3 & 20 & 1.85 & $-0.759438(2)$ \\
2 & $0.57(3)$ & -2.2 & 21 & 1.90 & $-0.755049(1)$ \\
3 & $0.59(2)$ & -2.1 & 22 & 1.95 & $-0.751498(2)$ \\
4 & $0.610(10)$ & -2.0 & 23 & 2.0 & $-0.748715(2)$ \\
5 & $0.634(4)$ & -1.9 & 24 & 2.1 & $-0.745199(1)$ \\
6 & $0.658(2)$ & -1.8 & 25 & 2.178 & $-0.744130(1)$ \\
7 & $0.6885(10)$ & -1.7 & 26 & 2.3 & $-0.744958(1)$ \\
8 & $0.7219(3)$ & -1.6 & 27 & 2.4 & $-0.747586(1)$ \\
9 & $0.7607(1)$ & -1.5 & 28 & 2.5 & $-0.751708(1)$ \\
10 & 0.8 & $-1.414(1)$ & 29 & 2.75 & $-0.7673233(4)$ \\
11 & 0.9 & $-1.2395(2)$ & 30 & 3.0 & $-0.7887774(4)$ \\
12 & 1.0 & $-1.11422(1)$ & 31 & 3.25 & $-0.8145143(3)$ \\
13 & 1.1 & $-1.02100(1)$ & 32 & 3.5 & $-0.8434661(2)$ \\
14 & 1.2 & $-0.95030(1)$ & 33 & 4.0 & $-0.9082113(2)$ \\
15 & 1.3 & $-0.896040(5)$ & 34 & 4.5 & $-0.9791030(2)$ \\
16 & 1.4 & $-0.854175(2)$ & 35 & 5.0 & $-1.0539340(2)$ \\
17 & 1.5 & $-0.821890(1)$ & 36 & 5.5 & $-1.1313743(2)$ \\
18 & 1.6 & $-0.797164(2)$ & 37 & 6.0 & $-1.2105830(3)$ \\
19 & 1.75 & $-0.771064(3)$ & & & \\
\hline \hline
\end{tabular}

for $l=L$ and $L+1$. These sequences appear to converge faster with increasing $L$ than the $K_{c}{ }^{(1)}(H, L)$. Remaining corrections may be due to additional contributions to Eq. (41), for instance scaling as $L^{2 y_{i}}$. Thus we defined $K_{c}^{(3)}(H, L)$ by solving the equation

$$
K_{c}^{(3)}(H, L)=K_{c}^{(2)}(H, l)+c_{2}(L) l^{2 y_{i}-y_{t}}
$$

for $l=L$ and $L+1$. Further estimates can be obtained with correction exponents $2 y_{i}-2 y_{t}$, or by treating the correction exponents as a free variable in which case three values of $l$ have to be used. Several variations of this procedure were tried which leads to some insight in the numerical inaccuracies of the fitting procedure.

Our final estimates of the critical points for $T>0$ are listed in Table I. The apparent accuracy of the critical points is satisfactory for most of the field range, but it deteriorates rapidly at small fields. Nevertheless this region has our special interest: we wish to determine how the Potts line connects to the KT point on the $T=0$ line, because this is where the theoretical predictions (see Secs. II and III) are markedly different.

We have also reconsidered the determination of the KT point at $T=0$ given in Ref. [12]. In that work, the finite-size data for the critical field $H_{\mathrm{KT}}$ were obtained by requiring that the scaled gap associated with the spin waves of period 6 , i.e., electric charges $e= \pm 1$ were equal to the expected value $X_{1,0}=2 / 9$ at the $\mathrm{KT}$ transition. These estimates $H_{\mathrm{KT}}(L)$ of the KT point, which were obtained for system sizes up to $L=27$, were found to be considerably size dependent. They were fitted according to $H_{\mathrm{KT}}(L)=H_{\mathrm{KT}}+a /(b+\ln L)+c L^{-2}$ which led to extrapolated estimates $H_{\mathrm{KT}}$ that displayed only a remarkably small size dependence. On this basis, the final

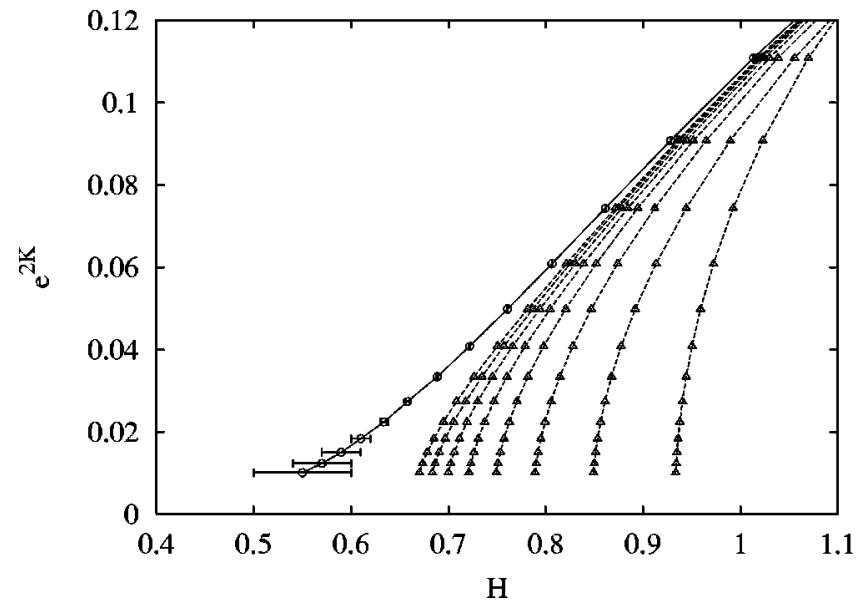

FIG. 3. Finite-size solutions for the critical points, and our final estimates in the region of small field and temperature. The dashed lines connect the solutions shown as triangles. From right to left the lines show data for finite sizes $L=3,6, \ldots, 24$. The solid line with circles indicates our final estimated result for the critical line.

estimate was given as $H_{\mathrm{KT}}=0.266 \pm 0.010$ in Ref. [12]. In our present work we have reproduced these data for $H_{\mathrm{KT}}(L)$. We have not used the procedure that requires that the scaled gap is equal for two subsequent system sizes. Since all points in the range $H \leqslant H_{\mathrm{KT}}$ satisfy this scaling equation asymptotically, the solutions may converge to any point in this range, depending on the corrections to scaling. This is another reason behind the discrepancy mentioned in Sec. I concerning an earlier result for the location of the KT point [15]. We have used the $H_{\mathrm{KT}}(L)$ data as input for several other iterated fit procedures consisting of subsequent extrapolation steps according to $L^{-2}$ behavior, and powers of $1 / \ln L$. These fits led to results for $H_{\mathrm{KT}}$ that were rather consistently close to 0.26 , with differences up to 0.02 .

As an independent approach we have estimated $H_{\mathrm{KT}}$ from the requirement that the scaled gap based on the magnetic dimension $X_{0,1 / 3}$ is equal to the expected value $1 / 8$ at $g_{R}$ $=9 / 4$. Since such fractional magnetic charges (corresponding with vortices of strength 2) do not exist in this model, this scaled gap cannot directly be calculated for a fixed system size $L$. However, it can be obtained by combining freeenergy data for system sizes $L=3 n \pm 1$, where $n$ is an integer, as explained in Ref. [12]. The same extrapolation procedures as above were tried, and led to results again consistent with $H_{\mathrm{KT}}=0.26$, but with differences up to about 0.04. Our final conclusion is $H_{\mathrm{KT}}=0.26 \pm 0.02$, similar to the value presented in Ref. [12] but with a slightly more conservative error estimate.

The numerical results for the critical points are combined in Fig. 1, the phase diagram in the $\left(H, e^{2 K}\right)$ plane. We remark that for large $|K|$ and relatively small $H$, the solutions for $H$ become strongly finite-size dependent and slowly convergent. This problem is apparently due to the proximity of the KT transition at $K=-\infty$. This is illustrated in Fig. 3, in which a set of lines represent the finite-size solutions for $L$ $=3,6, \ldots, 24$ together with the extrapolated critical line. On the basis of our limited range of finite system sizes, the es- 


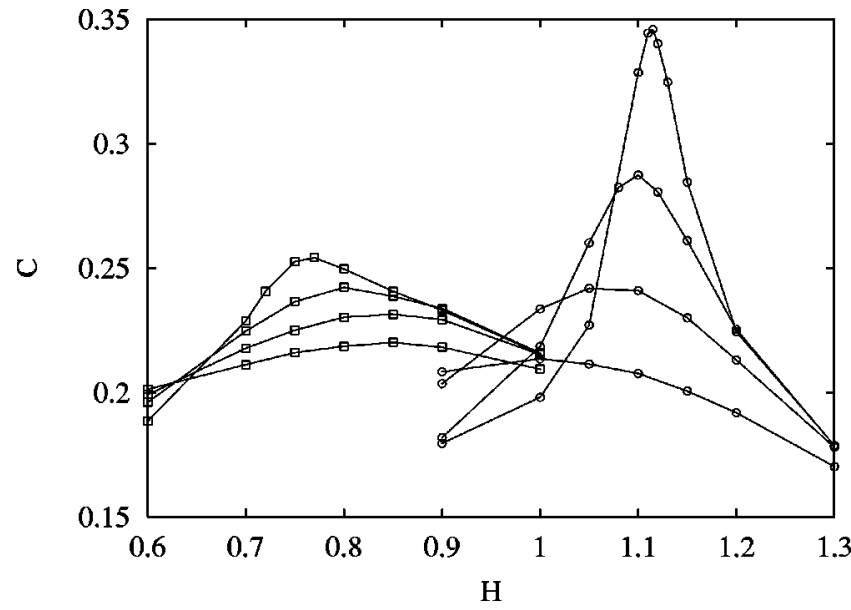

FIG. 4. Specific-heat-like quantity $C$ versus field $H$ for two values of the Ising coupling $K=-1.0(\bigcirc)$ and $-1.5(\square)$. Both sets of lines display data for system sizes $L=12,24,48$, and 96 . These two cases indicate that the amplitude of the divergence of $C$ decreases with decreasing field $H$.

timation of the critical points thus becomes increasingly difficult for large $|K|$.

In order to provide further justification for our assumption that the critical line belongs to the three-state Potts universality class (used for the determination of the critical points) we perform a consistency test by calculating the conformal anomaly $c$ at the estimated critical points at $H=1.5,2.5,3.5$, and 4.5. Iterated fits similar to those used for the calculation of the critical points were applied. All these results are consistent with the exact value $c=\frac{4}{5}$. The error margin varies between a few times $10^{-3}$ for $H=1.5$ and a few times $10^{-5}$ for $H=3.5$. In comparison with previous work [6], these results further restrict the scale of possible deviations from three-state Potts universality.

\section{B. Monte Carlo results}

The apparent difficulty to obtain accurate critical points for small $H$ by the transfer-matrix method invites further

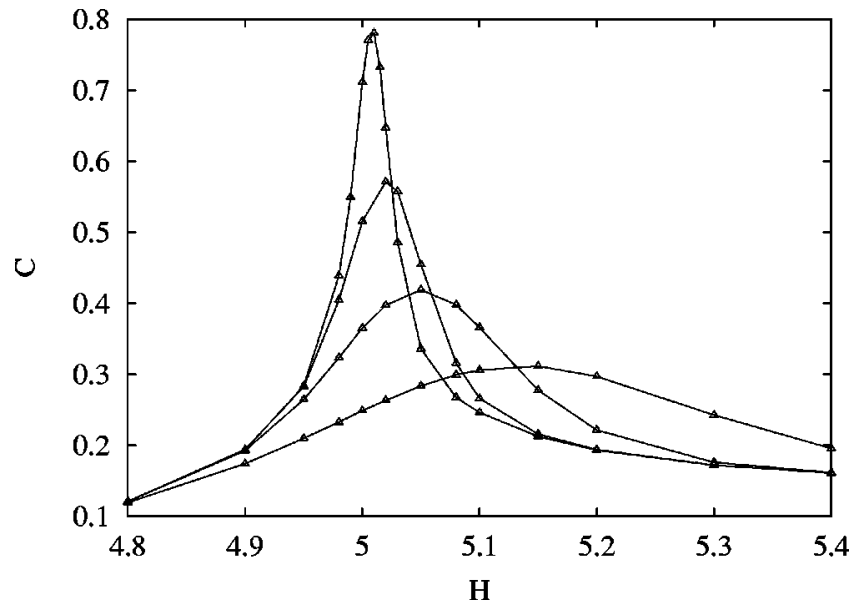

FIG. 5. Specific-heat-like quantity $C$ versus $H$ at Ising coupling $K=-1.053934$, near the critical point at $H=5.0$. In comparison with Fig. 4, the finite-size divergence is much stronger.

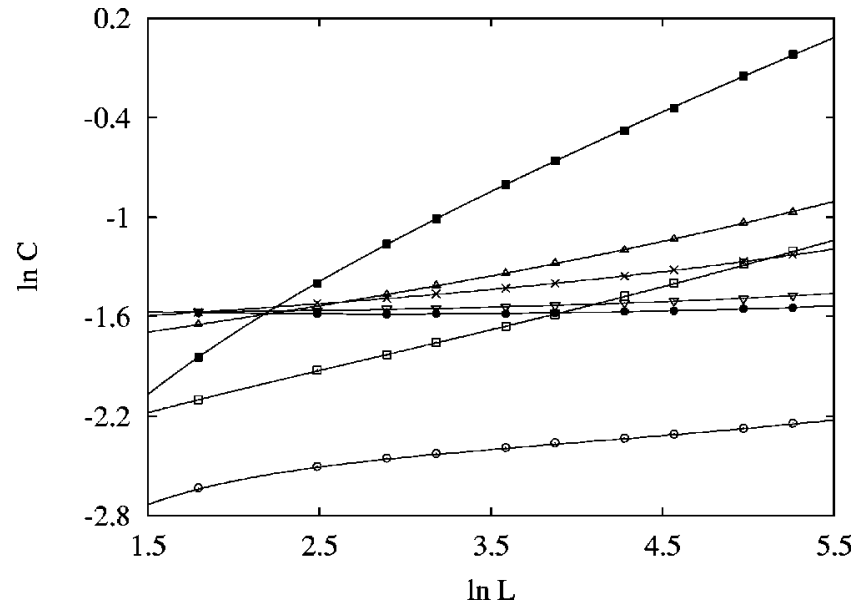

FIG. 6. Specific-heat-like quantity $C$ versus system size on a double logarithmic scale. Data are shown for system sizes $6 \leqslant L$ $\leqslant 192$ at seven points $(H, K)$ on the critical line. The symbol represents $H=0.61 ; \nabla: H=0.658 ; \times: H=0.8 ; \triangle: H=1.0 ; \square: H$ $=1.5 ; \bigcirc: H=2.0 ; \mathbf{\square}: H=5.0$. The seven lines represent the fitted results. The statistical errors are not shown in this figure. They do not exceed the thickness of the lines.

investigation by means of Monte Carlo simulations, which allow the use of much larger finite sizes. In particular we determine how the critical amplitudes behave for small $T$, and make a comparison with the renormalization prediction. To this purpose we define a specific-heat-like quantity $C$, i.e., the second order derivative of the free energy to a parameter conjugate to an energylike density in the Hamiltonian, for which we may take the magnetization. Indeed the Ising field $H$ drives the Potts-like transition to the ordered state (except at the maximum of the $T$ vs $H$ curve) and thus plays the role of the temperature in the Potts model. We thus define $C$ by

$$
C=\frac{\partial^{2} f}{\partial H^{2}}=N\left(\left\langle m^{2}\right\rangle-\langle m\rangle^{2}\right),
$$

where $m$ is the Ising magnetization. Similarly, we define a quantity similar to the magnetic susceptibility of the Potts model. In the $q=3$ Potts model, the zero-field magnetic susceptibility can be expressed in magnetization fluctuations by

$$
\chi=N\left\langle m_{P}^{2}\right\rangle
$$

where $m_{P}^{2}=n_{1}^{2}+n_{2}^{2}+n_{3}^{2}-n_{1} n_{2}-n_{2} n_{3}-n_{3} n_{1}$ expresses the Potts magnetization in terms of the densities $n_{i}$ of Potts variables in state $i$. In the scaling context of the present scaling analysis, the densities $n_{i}$ may be defined as the number of minus spins on sublattice $i$. Thus $\chi$ describes the response of the model to staggered fields acting on the Ising spins.

The simulations used triangular $L \times L$ lattices with periodic boundary conditions. We used a combination of the standard Metropolis algorithm and the geometric cluster method [28]. The latter method executes nonlocal updates and leads to a faster relaxation. But it does not change the Ising magnetization. For this reason also Metropolis steps 


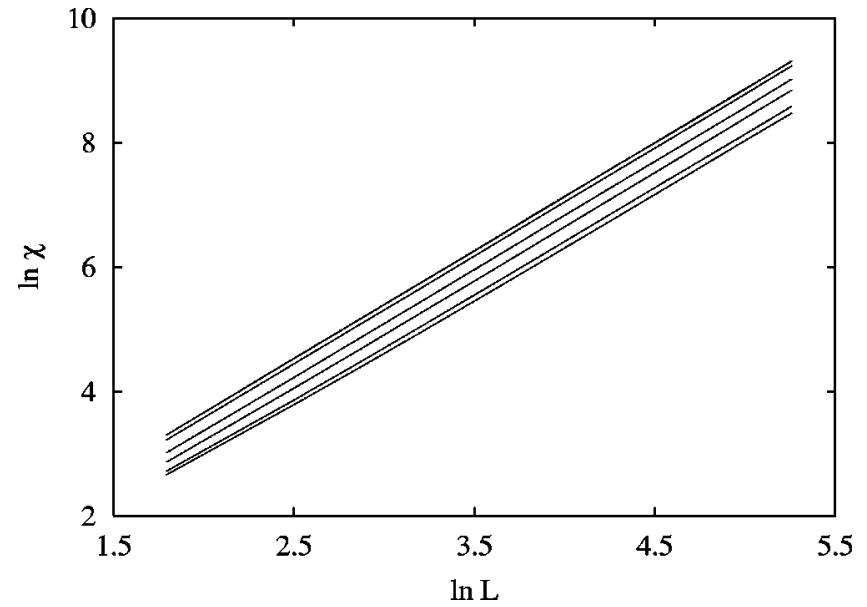

FIG. 7. Susceptibilitylike quantity $\chi$ versus system size $L$ $(=6, \ldots, 192)$ on a double logarithmic scale. Data are shown for seven points $(H, K)$ on the critical line. From bottom to top: $H$ $=0.61,0.658,0.8,1.0,1.5,2.0$, and 5.0, respectively. The two lines for $H=2.0$ and 5.0 coincide on this scale. The statistical errors are not shown in this figure. They do not exceed the thickness of the lines.

were included. First we sampled $C$ in a suitable range of $H$, to study its divergence, at fixed values of coupling $K$ which are taken from Table I. The results are shown in Figs. 4 and 5 and show that the finite-size divergence of $C$ at the critical line becomes weaker when $H$ decreases. In order to study this phenomenon in a more quantitative sense, we have determined $C$ and $\chi$ at several critical points taken from Table I for several system sizes. Results for $H=0.61,0.658,0.8$, 1.0, 1.5, 2.0, and 5.0 are shown in Figs. 6 and 7.

Finite-size scaling of the free energy density of a system with finite size $L$ can be expressed by

$$
f(t, h, u, L)=L^{-d} f\left(L^{y_{t}} t, L^{y_{h}} h, L^{y_{i}} u, 1\right)+g(t, h, u),
$$

where $t, h$, and $u$ denote the temperature, magnetic field, and irrelevant field, respectively, and $g$ the regular part of the transformation. Differentiation of $f$ yields the scaling behavior of the quantities $C$ and $\chi$ as

$$
\begin{aligned}
C(u, L) & =C_{0}+L^{2 y_{t}-d} C\left(L^{y_{i}} u, 1\right) \\
& =C_{0}+L^{2 y_{t}-d}\left(b_{0}+b_{1} L^{y_{i}} u+b_{2} L^{2 y_{i}} u^{2}+\cdots\right)
\end{aligned}
$$

and

$$
\begin{aligned}
\chi(u, L) & =\chi_{0}+L^{2 y_{h}-d} \chi\left(L^{y_{i}} u, 1\right) \\
& =\chi_{0}+L^{2 y_{h}-d}\left(b_{0}+b_{1} L^{y_{i}} u+b_{2} L^{2 y_{i}} u^{2}+\cdots\right) .
\end{aligned}
$$

We have fitted the numerical data by these two equations and thus derived the amplitudes listed in Tables II and III. The amplitude $b_{0}$ of the leading divergence of $C$ decreases with field except close to the maximum of the critical line in the $(H, T)$ plane. At the maximum the field $H$ fails to bring the system into the ordered phase and the amplitude $b_{0}$ thus
TABLE II. Parameters describing the finite-size behavior of the specific heatlike quantity $C$. The third column is the amplitude $b_{0}$ of the leading divergent term. The amplitudes for $H=1.5,2.0$ are relatively small because the critical line runs almost parallel to the field direction. The column corresponding with $b_{i}(i=1,2)$ are the irrelevant corrections amplitudes.

\begin{tabular}{lcccc}
\hline \hline$H$ & $C_{0}$ & $b_{0}$ & $b_{1}$ & $b_{2}$ \\
\hline 0.61 & $0.188(2)$ & $0.0026(2)$ & $0.027(3)$ & \\
0.658 & $0.196(2)$ & $0.0039(3)$ & $0.007(3)$ & \\
0.8 & $0.192(3)$ & $0.0126(4)$ & $-0.022(4)$ & \\
1.0 & $0.151(3)$ & $0.0285(6)$ & $-0.035(5)$ & \\
1.5 & $0.097(2)$ & $0.0254(4)$ & $-0.055(3)$ & \\
2.0 & $0.084(3)$ & $0.0029(3)$ & $-0.015(6)$ & $-0.097(2)$ \\
5.0 & $-0.096(6)$ & $0.131(2)$ & $-0.031(9)$ & \\
\hline \hline
\end{tabular}

vanishes. Also for the susceptibilitylike quantity $\chi$ the amplitude $b_{0}$ of the finite-size divergence, shown in Table III, decreases regularly when the KT point is approached.

The behavior of the amplitude $b_{0}$ at small field and low temperature follows from the renormalization-flow analysis. Starting from a point in the vicinity of the KT point, we renormalize until we arrive at the boundary with the region dominated by the three-state Potts fixed point. Let $b_{\mathrm{KT}}$ be the corresponding scale factor. Since the specific heatlike quantity $C$ is defined by means of differentiation of the free energy to the uniform field $H$, we keep track of how $H$ changes under this transformation. The marginality of $\delta h$ at $g_{R}$ $=9 / 4$ is expressed by Eq. (31): when we write $h=t+\delta h$, it is clear that $\delta h$ varies only by a factor of order 1 as long as $t$ is of order 1. In the context of scaling, we thus have $\delta h^{\prime}$ $\approx \delta h$, where the prime indicates the value at the boundary. Within the Potts region we rescale the system to size 1 with the remaining scale factor $L / b_{\mathrm{KT}}$

$$
\delta h^{\prime \prime}=\left(\frac{L}{b_{\mathrm{KT}}}\right)^{6 / 5} \delta h^{\prime} \approx L^{6 / 5} b_{\mathrm{KT}}^{-6 / 5} \delta h,
$$

where the Potts temperature exponent $\frac{6}{5}$ applies because it corresponds with the Ising magnetic field. The behavior of $C$ follows as

TABLE III. Parameters describing the finite-size behavior of the susceptibilitylike quantity $\chi$. The third column is the amplitude $b_{0}$ of the leading divergent term. It decreases regularly with the field. The next columns shows the irrelevant correction amplitudes $b_{i}(i$ $=1,2)$.

\begin{tabular}{lcccc}
\hline \hline$H$ & $\chi_{0}$ & $b_{0}$ & $b_{1}$ & $b_{2}$ \\
\hline 0.61 & $-17.5(18)$ & $0.5281(12)$ & $-0.143(50)$ & $16.3(16)$ \\
0.658 & $-10.5(20)$ & $0.5896(14)$ & $-0.142(55)$ & $2.88(36)$ \\
0.8 & $-18.7(37)$ & $0.7675(24)$ & $-0.75(10)$ & $18.1(33)$ \\
1.0 & $0.95(11)$ & $0.9046(17)$ & $-0.164(25)$ & \\
1.5 & $0.41(10)$ & $1.1209(12)$ & $-0.103(24)$ & \\
2.0 & $0.10(14)$ & $1.2130(24)$ & $-0.003(34)$ & \\
5.0 & $-0.51(14)$ & $1.2133(22)$ & $0.070(37)$ & \\
\hline \hline
\end{tabular}




$$
C=\frac{\partial^{2} f}{\partial h^{2}}=L^{-d} \frac{\partial^{2}}{\partial h^{2}} f\left(L^{6 / 5} b_{\mathrm{KT}}^{-6 / 5} h, 1\right)=L^{2 / 5} b_{\mathrm{KT}}^{-12 / 5} f^{\prime \prime}+\text { const. }
$$

The susceptibilitylike quantity $\chi$ is obtained by differentiation of $f$ to the staggered Ising field, which, as explained in Ref. [10], is associated with a Gaussian spin wave perturbation of period $p=6$, i.e., with electric charges $e= \pm 1$. The exponent of the staggered field thus takes the value $2-X_{1,0}$ $=16 / 9$ at the KT fixed point. Therefore, at the boundary with the Potts region we have

$$
h_{\mathrm{st}}^{\prime}=b_{\mathrm{KT}}^{16 / 9} h_{\mathrm{st}} .
$$

Within the region dominated by the Potts fixed point, the magnetic exponent $y_{m}=28 / 15$ applies. Renormalization with the remaining scale factor $L / b_{\mathrm{KT}}$ leads to

$$
h_{\mathrm{st}}^{\prime \prime}=\left(\frac{L}{b_{\mathrm{KT}}}\right)^{28 / 15} h_{\mathrm{st}}^{\prime}=L^{28 / 15} b_{\mathrm{KT}}^{-4 / 45} h_{\mathrm{st}}
$$

so that $\chi$ scales as

$$
\begin{aligned}
\chi & =\frac{\partial^{2} f}{\partial h_{\mathrm{st}}^{2}}=L^{-d} \frac{\partial^{2}}{\partial h_{\mathrm{st}}^{2}} f\left(L^{28 / 15} b_{\mathrm{KT}}^{-4 / 45} h_{\mathrm{st}}, 1\right) \\
& =L^{26 / 15} b_{\mathrm{KT}}^{-8 / 45} f^{\prime \prime}+\text { const. }
\end{aligned}
$$

According to Sec. III B, rescaling by a factor $b_{\mathrm{KT}}$ results in an Ising temperature field $b_{\mathrm{KT}}^{7 / 8} e^{2 K}=\beta / 2$ so that $b_{\mathrm{KT}}$ $\propto e^{-(16 / 7) K}$. For strong coupling the renormalization scale $b_{\mathrm{KT}}$ is large, which is indicative of the crossover phenomenon close to the KT transition. There we need large system sizes $L>b_{\mathrm{KT}}$ in order to reach the vicinity of the Potts fixed point. The substitution of $b_{\mathrm{KT}}$ into Eq. (52) and Eq. (55), leads to

$$
C=L^{2 / 5} e^{(192 / 35) K} f^{\prime \prime}+\text { const }
$$

and

$$
\chi=L^{26 / 15} e^{(128 / 315) K} f^{\prime \prime}+\text { const. }
$$

From a comparison of Eqs. (49) and (56), and of Eqs. (50) and (57), we expect that $b_{0} \propto e^{(192 / 35) K}$ for $C$, and $b_{0}$ $\propto e^{(128 / 315) K}$ for $\chi$, when $|K|$ is large enough. We thus expect a linear relation between $\ln b_{0}$ and $K$ for sufficiently strong coupling $K$. A fit to the numerical data yields the slopes as about 2.8(1) for $C$ and 0.64(4) for $\chi$. These slopes do not agree accurately with the analytic values. This suggests that the Ising temperature used for the calculation of the amplitudes is not small enough. However, the qualitative amplitude dependence is reproduced, and the rough agreement suggests that we are not far away from the asymptotic regime.
TABLE IV. Coefficients $\kappa_{i}^{j}(i=0, \ldots, 6 ; j=1,2,3)$ of the invariant polynomial $f_{6}$, Eq. (19). The condition for criticality reads $f_{6}$ $=0$.

\begin{tabular}{ccc}
\hline \hline$\kappa_{0}^{1}$ & $\kappa_{1}^{1}$ & $\kappa_{2}^{1}$ \\
-0.002942307242 & -0.097656582607 & 2.725303204552 \\
$\kappa_{0}^{2}$ & $\kappa_{1}^{2}$ & $\kappa_{2}^{2}$ \\
-0.000000000017 & 0.000351055639 & -0.020534345140 \\
$\kappa_{3}^{2}$ & $\kappa_{4}^{2}$ & $\kappa_{3}^{3}$ \\
0.044511873969 & 0.660086779714 & 0.000001077354 \\
$\kappa_{4}^{3}$ & $\kappa_{5}^{3}$ & $\kappa_{6}^{3}$ \\
-0.002865984972 & -0.084578467922 & 1.000000000000 \\
\hline \hline
\end{tabular}

\section{FIT AND DISCUSSION}

\section{A. Roots of the $\mathbf{O ( 2 )}$ invariant polynomials}

The number of 37 critical points in Table I together with the additional KT point $(h, z)=\left(h_{\mathrm{KT}},-1\right)$ is sufficient to attempt fits of $\mathrm{O}(2)$ invariant polynomials 19 up to order 10. We have not used data points 1 and 2 because of their limited accuracy, and performed a least-squares fit to the remaining 35 data points for $z>-1$. We have also tried direct fits to several subsets of these. In each case we have investigated the effect of enforcing the curve to pass through the KT point, or to extract the value of $h_{\mathrm{KT}}$ from the fit. It is found that the coefficients in the equation $f_{4}=0$ are not flexible enough to even qualitatively fit the numerical data. The leastsquares fit to $f_{6}=0$ excluding the KT point consists of two avoiding solutions, which lead to 2 disconnected "critical" lines which have unphysical ranges. For $z=-1$ one line terminates at $h^{*} \approx 0.501>h_{\mathrm{KT}}$. When enforcing the $\mathrm{KT}$ point at $h_{\mathrm{KT}}=0.25$, the two avoiding branches repel one another even stronger. Direct fits to different subsets of critical

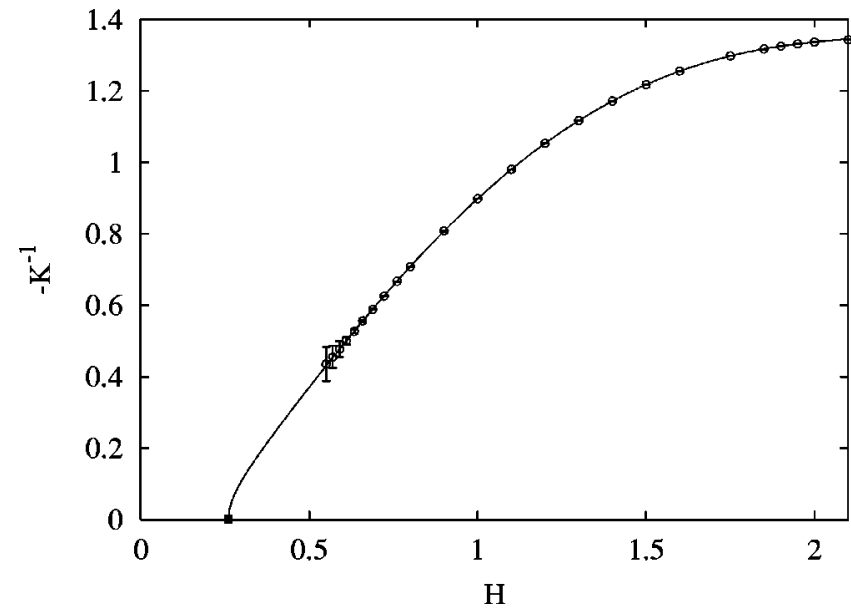

FIG. 8. Phase diagram in the $\left(H, K^{-1}\right)$ plane. The symbol $\bigcirc$ denotes the Potts transition points; the KT point; and the solid line describes the fit of the expression based on the renormalization prediction for the critical line in the small field region. 
TABLE V. Coefficients $a_{j}$ of the expansion (58) of $-K^{-1}$ in powers of $\left(H-H_{\mathrm{KT}}\right)^{j / 2}$.

\begin{tabular}{ccc}
\hline \hline$a_{1}$ & $a_{2}$ & $a_{3}$ \\
0.487432 & 0.119116 & 0.765066 \\
& & $a_{6}$ \\
$a_{4}$ & $a_{5}$ & 0.652161 \\
1.017104 & -1.949253 & \\
\hline \hline
\end{tabular}

points lead to similar results. Fits to higher order equations $f_{8}=0$ and $f_{10}=0$ display the same problems. Even more avoiding solutions enter. The numerical problems are clearly displayed by the values of the fitted coefficients, which span a range of many orders of magnitude. In summary, the roots of invariant polynomial equations cannot fit the critical curve. The main problem is the approach of the curve to the KT limit imposed by Eq. (12): all such roots approach the KT point vertically in the $(h, z)$ plane, whereas the numerical data in Fig. 9 indicate a horizontal approach.

Sufficiently far away from the KT point, the problem dissolves, and our numerical data for the critical points can well be approximated by means of invariant polynomials. For example, the polynomial of order 6 can reproduce the critical points for $H \geqslant 1.5$ within the error margins quoted in Table I. The coefficients, determined by means of a least-squares fit, are listed in Table IV.

\section{B. The renormalization solution for small field}

For small field we expand Eq. (37) and take into account higher order terms in the physical fields. This leads to

$$
-\frac{1}{K}=\sum_{j=1,2, \ldots} a_{j}\left(H-H_{\mathrm{KT}}\right)^{j / 2} .
$$

The numerical data for the critical points for $H \leqslant 1.75$ are fitted satisfactorily (i.e., within the error margins quoted in Table I) by this formula using six coefficients. The numerical results and the fitted function are shown in Fig. 8. The values of the coefficients are listed in Table V.

\section{CONCLUSION}

The invariant-polynomial scenario formulated in Sec. II and the renormalization scenario formulated in Sec. III lead to analytic expressions for the critical line in the $(H, T)$ diagram that are mutually inconsistent for $T \downarrow 0$ at finite $H$. This shows that at least one of the underlying assumptions must be incorrect. The renormalization prediction appears to successfully describe the numerical data for small $|H|$. Although the asymptotic regime is not quite reached (as can, for instance, be seen in Fig. 8 where the leftmost points behave almost linearly instead of as a square root), an asymptotic expansion leads to an accurate description of the data, and allows a smooth extrapolation to zero Ising temperature in agreement with Eq. (37). The analysis in Sec. IV B of the

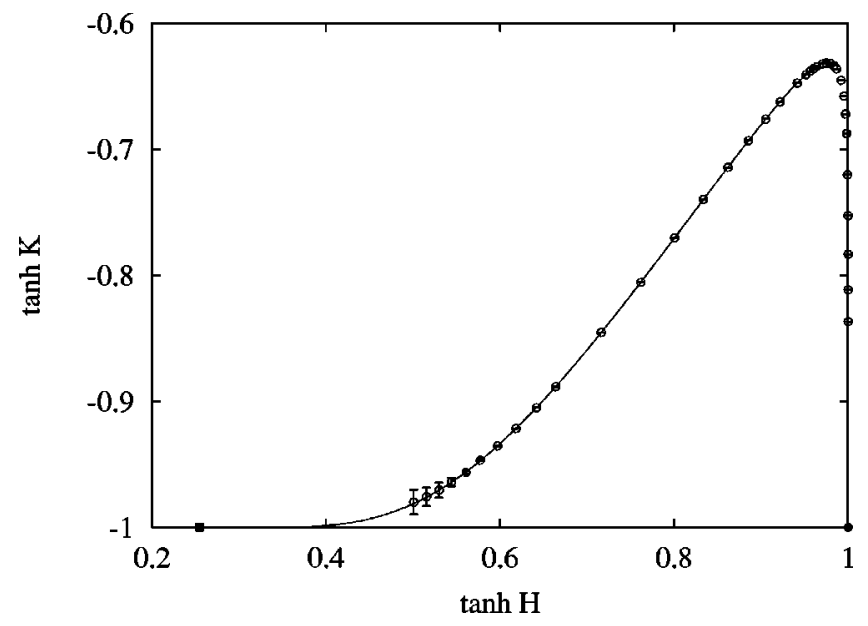

FIG. 9. Phase diagram in the $(h, z)$ plane (where $h=\tanh H$ and $z=\tanh K$ ). The KT point is denoted as $\mathbf{\square}$, and the LG point, which is Baxter's hard-hexagon model, as

critical amplitudes fits precisely in this picture. Thus our analysis does not give reasons to doubt that the renormalization scenario correctly describes the essential physics of the model near the KT transition.

In contrast, the invariant-polynomial scenario does not agree with the numerical data. It predicts a "vertical" approach to the KT point in the $(h, z)$ diagram (see Fig. 9) where it should be horizontal. Our interpretation is that the assumption of analyticity of the critical line in the $(h, z)$ parametrization is false at the KT point, so that the line cannot be described by the zeroes of a polynomial of a finite order.

Since it now appears that the invariant-polynomial scenario fails in the case of the triangular-lattice Ising model, the question arises whether similar, apparently successful, analyses of the critical lines of the honeycomb- and the square-lattice Ising model in terms of invariant polynomials $[19,20]$ have to be reconsidered. Here we may point at the simpler topology of the $(H, T)$ diagram for the honeycomb and the square lattices: the critical line connects to $T=0$ only in the lattice-gas points $H= \pm \infty$. In the case of the triangular lattice model, crossover phenomena near the KT point are responsible for the nonanalytic "shape" of the critical line. In the absence of such crossover phenomena, there is no inconsistency with the invariant-polynomial scenario, and our present analysis has therefore no direct consequences for the work presented in Refs. [19,20].

\section{ACKNOWLEDGMENTS}

We are indebted to Professor Fa Y. Wu and Professor Bernard Nienhuis for several illuminating discussions and useful conversations, to Dr. Xue-Ning Wu for her valuable contributions in the earlier stages of this project, and to Dr. Jouke R. Heringa for his contribution to the development of the geometric cluster algorithm used in this work. 
[1] R.M.F. Houtappel, Physica (Amsterdam) 16, 425 (1950).

[2] J. Stephenson, J. Math. Phys. 11, 413 (1970).

[3] S. Alexander, Phys. Lett. 54A, 353 (1975).

[4] W. Kinzel and M. Schick, Phys. Rev. B 23, 3435 (1981).

[5] M.P. Nightingale, Proc. K. Ned. Akad. Wet., Ser. B: Palaeontol., Geol., Phys., Chem., Anthropol. 82, 235 (1979).

[6] J.D. Noh and D. Kim, Int. J. Mod. Phys. B 6, 2913 (1992).

[7] M.N. Tamashiro and S.R. Salinas, Phys. Rev. B 56, 8241 (1997).

[8] Z. Ràcz, Phys. Rev. B 21, 4012 (1980).

[9] R.J. Baxter, Exactly Solved Models in Statistical Physics (Academic Press, New York, 1982).

[10] B. Nienhuis, H.J. Hilhorst, and H.W.J. Blöte, J. Phys. A 17, 3559 (1984).

[11] H.W.J. Blöte and H.J. Hilhorst, J. Phys. A 15, L631 (1982).

[12] H.W.J. Blöte and M.P. Nightingale, Phys. Rev. B 47, 15046 (1993).

[13] J.M. Kosterlitz and D.J. Thouless, J. Phys. C 5, L124 (1973); 6, 1181 (1973).

[14] H.W.J. Blöte, M.P. Nightingale, X.N. Wu, and A. Hoogland, Phys. Rev. B 43, 8751 (1991).

[15] S.L.A. de Queiroz, T. Paiva, J.S. de Sá Martins, and R.R. dos Santos, Phys. Rev. E 59, 2772 (1999).

[16] S.L.A. de Queiroz (private communication). The extrapolated slope $S_{L}$ in Table I of the preceding reference is thus the inverse of our $H_{\mathrm{KT}}$.

[17] F.Y. Wu, J. Math. Phys. 15, 687 (1974).

[18] F.Y. Wu and X.N. Wu, J. Stat. Phys. 90, 41 (1988).

[19] F.Y. Wu, X.N. Wu, and H.W.J. Blöte, Phys. Rev. Lett. 62, 2773 (1989).

[20] H.W.J. Blöte and X.N. Wu, J. Phys. A 23, L627 (1990).

[21] J.H.H. Perk, F.Y. Wu, and X.N. Wu, J. Phys. A 23, L131 (1990).

[22] J.V. José, L.P. Kadanoff, S. Kirkpatrick, and D.R. Nelson, Phys. Rev. B 16, 1217 (1977).

[23] B. Nienhuis, in Phase Transitions and Critical Phenomena edited by C. Domb and J.L. Lebowitz (Academic, London, 1987), Vol. 11.

[24] X.F. Qian and H.W.J. Blöte (unpublished). We have introduced next-nearest-neighbor interactions $K_{\mathrm{NNN}}$ and used a transfermatrix method to locate the $g_{R}=3$ point and the approximate location of the Potts critical line for that value of $K_{\mathrm{NNN}}$.

[25] D.P. Landau, Phys. Rev. B 27, 5604 (1983).

[26] H.W.J. Blöte, F.Y. Wu, and X.N. Wu, Int. J. Mod. Phys. B 4, 619 (1990).

[27] J.L. Cardy, J. Phys. A 17, L358 (1984).

[28] J.R. Heringa and H.W.J. Blöte, Phys. Rev. E 57, 4976 (1998). 